\title{
Are women with small endometriomas who undergo intracytoplasmic sperm injection at an elevated risk for adverse pregnancy, obstetric, and neonatal outcomes?
}

\author{
Fatma Ferda Verit ${ }^{1}$, Ayse Seyma Ozsuer Kucukakca ${ }^{2}$ \\ 'Department of Obstetrics and Gynecology, University of Health Sciences, Istanbul Training and Research Hospital, Istanbul; ${ }^{2}$ Department of \\ Obstetrics and Gynecology, Bezmialem Foundation University, Istanbul, Turkey
}

Objective: The aim of the study was to investigate pregnancy, obstetric, and neonatal outcomes in women with small $(<4 \mathrm{~cm})$ unilateral endometriomas.

Methods: This retrospective study included 177 patients: 91 patients with small endometriomas and 86 controls with unexplained or tubal factor infertility who were treated at the Süleymaniye Gynecology and Maternity Training and Research Hospital Infertility Unit between January 2010 and July 2015. The groups were matched with regards to demographic characteristics such as age, body mass index, and infertility duration. All of the women in this study conceived via intracytoplasmic sperm injection. We compared pregnancy, obstetric, and neonatal outcomes between these groups.

Results: Women with endometriomas had a higher biochemical pregnancy rate, but lower clinical pregnancy and live birth rates than women with unexplained and tubal factor infertility ( $p<0.05$ for all). However no significant differences were found in terms of obstetric and neonatal complications between the two groups ( $p>0.05$ for all).

Conclusion: In this study, we found that women with endometriomas less than $4 \mathrm{~cm}$ were more prone to early pregnancy complications. We also showed that this group did not have any increased risks of late pregnancy, obstetric, and neonatal complications.

Keywords: Endometrioma; Neonatal; Obstetric outcome; Pregnancy

\section{Introduction}

Endometriosis is a chronic, estrogen-dependent, inflammatory disease that is associated with pelvic pain and infertility. Approxi-

Received: April 17, 2020 · Revised: July 27, 2020 · Accepted: August 20, 2020 Corresponding author: Fatma Ferda Verit

Department of Obstetrics and Gynecology, Istanbul Training and Research Hospital, Kasap Ilyas Mah. Org. Abdurrahman Nafiz Gurman Cd., TR-34098 Fatih/ Istanbul, Turkey

Tel: +90-212-4596000 Fax: +90-212-4596230 E-mail: fverit@gmail.com

This is an Open Access article distributed under the terms of the Creative Commons Attribution Non-Commercial License (http://creativecommons.org/licenses/by-nc/4.0/) which permits unrestricted non-commercial use, distribution, and reproduction in any medium, provided the original work is properly cited. mately $30 \%-50 \%$ of women with endometriosis have infertility problems, most of whom seek to achieve pregnancy through assisted reproductive technology (ART) [1]. Although the exact pathogenesis of infertility in these patients remains unclear, studies have proposed that endometriosis may be associated with some potential etiological factors, such as poor ovarian reserve, poor oocyte and embryo quality, endometrial dysfunction, implantation defects, increased inflammation in the peritoneal fluid, distorted pelvic anatomy, and adhesions.

Studies have also demonstrated that the eutopic endometrium of women with endometriosis has some problems such as stem cell content, hormonal sensitivity, junctional zone defects, and the pres- 
ence of proliferation and adhesion-inducing molecules that may impair implantation and decidualization [2]. Although it has been suggested that ovarian endometrioma, peritoneal endometriosis, and deep infiltrating endometriosis may have distinct molecular pathologies, similar endometrial changes are observed in all three of these conditions [3]. However, the outcomes may be worse at more advanced stages of the disease [4].

Conflicting data have been reported regarding the relationship between endometriosis and poor pregnancy outcomes. Some studies suggested that the incidence of placentation defects due to impaired decidualization such as preeclampsia, preterm birth, small for gestational age, and placenta previa may be higher in women with endometriosis $[5,6]$, but other studies reported no such associations [7-9]. These discrepancies may be due to considerable variation across studies in methodology, design, sample size, study population, the subtype of endometriosis, and diagnostic accuracy. Confounders such as coexisting adenomyosis cannot be totally excluded in observational studies. The selection of control groups and subgroup analyses are also matters of debate. Moreover, pregnancies in these women are mostly achieved through ART, which may have negative effects on pregnancy and neonatal outcomes.

Some studies have investigated the relationships between ovarian endometrioma and pregnancy outcomes; however the endometriomas included in these studies were heterogeneous in size and subgroup analyzes were not performed $[9,10]$. It is unknown whether the size of the endometrioma may also be an important factor affecting these outcomes. It is possible that outcomes may be worse in patients with more advanced disease, and patients must be informed of this possibility before conception [4]. The aim of the study was to investigate pregnancy, obstetric, and neonatal outcomes in pregnancies achieved through intracytoplasmic sperm injection (ICSI) in women with endometriomas smaller than $4 \mathrm{~cm}$.

\section{Methods}

The study was approved by the Institutional Review Board of Süleymaniye Gynecology and Maternity Training and Research Hospital (IRB No. 02-2015). Owing to the retrospective design, the requirement for informed consent was waived.

This retrospective study was performed at the Süleymaniye Gynecology and Maternity Training and Research Hospital Infertility Unit and included patients who met our eligibility criteria between January 2010 and July 2015. The study included 177 patients: 91 with small $(<4 \mathrm{~cm})$ unilateral endometriomas and 86 who did not have endometriomas and were diagnosed with unexplained or tubal factor infertility. Controls were matched to endometrioma patients in terms of demographic characteristics such as age, body mass index
(BMI), and infertility duration. We compared pregnancy, obstetric, and neonatal outcomes in these groups. Data were collected using patients' charts from our in vitro fertilization (IVF), obstetric, and neonatal intensive care unit. Patients were also contacted by telephone to gather any missing data about the outcomes. Women who were managed throughout their pregnancy by other obstetric units were not included.

Ovarian endometrioma was suspected based on the presence of a round cystic mass with a minimum diameter of $1 \mathrm{~cm}$ with thick walls, homogeneous fluid, and scattered internal echoes on ultrasonography [11]. The presence of suspected endometriomas was documented on at least one previous ultrasound examination at least 2 months before the ART cycle. The diameters of the suspected endometriomas were calculated using the mean values of three perpendicular layers. Doubtful and atypical cases were excluded. Conception was achieved by ICSI in all of the women in the study. The patients were treated using the standardized clinical protocol of our IVF unit. Only cases with fresh embryo transfers were included. Pregnancy was confirmed by a plasma beta-human chorionic gonadotropin ( $\beta$-hCG) level of $>20 \mathrm{IU} / \mathrm{L}$ at 14 days after oocyte retrieval.

The pregnancy outcomes included chemical pregnancy, miscarriage (spontaneous pregnancy loss at $<20$ weeks), pregnancy-induced hypertension (gestational hypertension, preeclampsia), preterm birth (delivery at $<37$ weeks of gestation), small for gestational age (birth weight $<10$ th percentile), placenta previa (placenta covering the cervix partially or completely), gestational diabetes, intrauterine growth restriction (birth weight $<10$ th percentile because of a pathological process; the fetus has not attained its biologically determined growth potential).

Biochemical pregnancy was defined as a very early miscarriage with a transient increase in serum hCG levels before the fifth week of gestation and before the fetus was visible on ultrasound. Preeclampsia was defined as a blood pressure reading $\geq 140 / 90 \mathrm{mmHg}$ after 20 weeks of gestation with proteinuria in formerly normotensive women. Gestational hypertension was defined as an elevated blood pressure reading ( $\geq 140 / 90 \mathrm{mmHg}$ ) after 20 weeks of gestation without proteinuria in previously normotensive women. Gestational diabetes was defined as carbohydrate intolerance with onset or recognition in pregnancy based on a positive oral glucose tolerance test. Data about neonates were collected after their admission to the neonatal intensive care unit.

The primary exclusion criteria were age above 37 years; endometrioma larger than $4 \mathrm{~cm}$; prior cystectomy or any surgery that may affect ovarian reserve; bilateral endometriomas; adenomyosis; intramural myomas; uterine malformations; polycystic ovarian disease; obesity (BMI $\geq 30 \mathrm{~kg} / \mathrm{m}^{2}$ ); chronic diseases such as hypertension, cardiovascular disease, diabetes mellitus, insulin resistance, hyperlip- 
idemia, liver or kidney disease, and neoplastic disease; a history of venous thromboembolism, antiphospholipid syndrome, or poor obstetric outcomes; ectopic pregnancy; hydrosalpinx; multiple pregnancies; autoimmune disease; smoking; and alcohol consumption. The neonatal outcomes analyzed were factors associated with increased neonatal morbidity and mortality such as perinatal asphyxia, necrotizing enterocolitis, congenital abnormalities, hypoglycemia, jaundice, hypothermia, and sepsis.

\section{Statistical analysis}

Data are presented as percentages for categorical variables and means for continuous variables. Proportions were compared using the Fisher exact test or chi-square test. Continuous variables were analyzed using the Student $t$-test or the Mann-Whitney $U$-test. For all tests, $p$-values of $<0.05$ were considered to indicate statistical significance.

\section{Results}

The baseline characteristics of the study and control groups are summarized in Table 1. There were no differences in demographic characteristics such as age, BMI, duration of infertility, and previous pregnancies between the groups. However, day 3 follicle-stimulating hormone and anti-Müllerian hormone levels were significantly dif-

Table 1. Baseline characteristics of the patients

\begin{tabular}{lccc}
\hline Variable & $\begin{array}{c}\text { Endometrioma } \\
(\mathrm{n}=91)\end{array}$ & $\begin{array}{c}\text { No endometrioma } \\
(\mathrm{n}=86)\end{array}$ & $p$-value \\
\hline Age $(\mathrm{yr})$ & $31.4 \pm 3.8$ & $30.4 \pm 3.5$ & 0.071 \\
BMl $\left(\mathrm{kg} / \mathrm{m}^{2}\right)$ & $24.3 \pm 3.7$ & $24.3 \pm 3.2$ & 0.95 \\
Duration of infertility $(\mathrm{yr})$ & $6.9 \pm 3.3$ & $6.2 \pm 3.3$ & 0.17 \\
Previous pregnancy & $11(12.1)$ & $14(16.3)$ & 0.42 \\
Day 3 FSH $(\mathrm{mlU} / \mathrm{mL})$ & $7.3 \pm 3.1$ & $6.2 \pm 2.0$ & 0.005 \\
AMH $(\mathrm{ng} / \mathrm{mL})$ & $2.1 \pm 1.3$ & $3.0 \pm 2.3$ & 0.002 \\
\hline
\end{tabular}

Values are presented as mean \pm standard deviation or number (\%). $\mathrm{BMI}$, body mass index; FSH, follicle-stimulating hormone; $\mathrm{AMH}$, antiMüllerian hormone.

Table 2. Treatment cycle characteristics

\begin{tabular}{lccc}
\hline Variable & $\begin{array}{c}\text { Endometrioma } \\
(\mathrm{n}=91)\end{array}$ & $\begin{array}{c}\text { No endometrioma } \\
(\mathrm{n}=86)\end{array}$ & $p$-value \\
\hline Total FSH dose (IU) & $2,685.5 \pm 798.5$ & $2,076.0 \pm 752.3$ & $<0.001$ \\
Day of stimulation & $10.3 \pm 1.7$ & $9.8 \pm 1.1$ & 0.028 \\
Number of oocytes retrieved & $6.0 \pm 4.3$ & $8.4 \pm 4.5$ & $<0.001$ \\
Number of transferred & $1.3 \pm 0.5$ & $1.3 \pm 0.4$ & 0.71 \\
$\quad$ embryos & & & \\
\hline
\end{tabular}

Values are presented as mean \pm standard deviation. FSH, follicle-stimulating hormone. ferent in the endometrioma group.

Treatment cycle characteristics are shown in Table 2. Women with endometriomas received higher doses of gonadotropins, the duration of stimulation was longer, and fewer oocytes were retrieved. No significant differences were found in terms of transferred embryos between the groups.

Pregnancy and neonatal outcomes are shown in Table 3. There were no differences in terms of the number of pregnancies, miscarriage, obstetric complications such as preterm birth, small for gestational age births, intrauterine growth restriction, preeclampsia, placenta previa, and gestational diabetes. No significant differences were also found in neonatal complications such as asphyxia, necrotizing enterocolitis, hyperbilirubinemia, and stillbirth. Nonetheless, the endometrioma group had a higher biochemical pregnancy rate and lower clinical pregnancy and live birth rates.

\section{Discussion}

The aim of the study was to evaluate the relationship between unilateral small ovarian endometriomas $(<4 \mathrm{~cm}$ ) and pregnancy, obstetric, and neonatal outcomes. We demonstrated that women with endometriomas less than $4 \mathrm{~cm}$ who underwent ICSI had poor pregnancy outcomes, such as a higher biochemical pregnancy rate and lower clinical pregnancy and live birth rates than ICSI control patients with unexplained or tubal factor infertility. However, women

Table 3. Pregnancy and neonatal outcomes

\begin{tabular}{lccc}
\hline Variable & $\begin{array}{c}\text { Endometrioma } \\
(\mathrm{n}=91)\end{array}$ & $\begin{array}{c}\text { No endometrioma } \\
(\mathrm{n}=86)\end{array}$ & $p$-value \\
\hline Number of pregnancies & $45(49.5)$ & $46(53.5)$ & 0.60 \\
Biochemical pregnancy & $14(31.1)$ & $2(4.3)$ & $<0.001$ \\
Clinical pregnancy & $31(68.9)$ & $44(95.7)$ & 0.001 \\
Miscarriage & $5(11.1)$ & $6(13.0)$ & 0.77 \\
Live birth & $26(57.8)$ & $38(82.6)$ & 0.01 \\
Obstetric complication & $6(23.1)$ & $4(10.5)$ & 0.17 \\
Preterm birth & $6(23.1)$ & $9(23.7)$ & 0.95 \\
SGA birth & $2(7.7)$ & $2(5.3)$ & 0.69 \\
IUGR & $1(3.8)$ & 0 & 0.35 \\
Preeclampsia & $1(3.8)$ & 0 & 0.22 \\
Placenta previa & $2(7.7)$ & $1(2.6)$ & 0.34 \\
Gestational diabetes & $1(3.8)$ & 0 & 0.22 \\
Neonatal complication & $3(11.5)$ & $2(5.3)$ & 0.35 \\
Asphyxia & $1(3.8)$ & $1(2.6)$ & 0.78 \\
Necrotizing enterocolitis & $1(3.8)$ & 0 & 0.22 \\
Hyperbilirubinemia & $1(3.8)$ & $1(2.6)$ & 0.78 \\
Stillbirth & 0 & 0 & \\
\hline
\end{tabular}

Values are presented as number (\%).

SGA, small for gestational age; IUGR, intrauterine growth restriction. 
with endometriomas did not have higher risks of obstetric and neonatal complications in the study.

In our study, we demonstrated that endometriomas smaller than $4 \mathrm{~cm}$ were associated with poor pregnancy outcomes. The rate of biochemical pregnancies was higher, while the clinical pregnancy and live birth rates were lower in patients with endometriomas; however, the miscarriage rate did not differ between the endometrioma and control groups. Although many studies have investigated the effect of endometriosis on pregnancy outcomes, the evidence is still conflicting [12-16]. Some studies reported that women with endometriosis did not have different clinical pregnancy, miscarriage, and live birth rates from their healthy counterparts $[12,13]$ while others found lower clinical pregnancy and live birth rates in endometriosis patients [14-16]. Omland et al. [17] also demonstrated that abortions prior to 6 weeks were more common and live birth rates were lower in women undergoing IVF with endometriosis than in those with unexplained infertility. However, the miscarriage rate after 6 weeks was not significantly different between the groups [17]. The low live birth rates in our study were associated with a higher biochemical pregnancy rate and a lower clinical pregnancy rates, but not with obstetric and perinatal complications.

What is the relationship between poor pregnancy outcomes and small endometriomas? There is considerable evidence that endometriosis may negatively affect follicle, oocyte, and embryo quality, as well as embryogenesis and embryo viability [12]. We suggest that endometriosis has a significant adverse environmental impact, which oocytes and embryos may not be able to tolerate in the early stages of pregnancy. Inflammatory mediators such as macrophages, cytokines, and vasoactive substances and oxidative stress cannot be blocked by IVF or ICSI in patients with endometriosis. It was reported that the fertilization capacity of oocytes and the developmental potential of embryos decreased when oocytes and embryos were cultured in media with peritoneal fluid obtained from women with endometriosis [18]. Inflammation within the ovary and endometriotic peritoneal fluid may impair oocyte and embryo development by blocking embryo growth factor receptor/signal transduction [19]. The inflammatory peritoneal fluid of women with endometriosis may also be toxic to the endometrium and the preimplantation embryo [20]. It has been also demonstrated that the eutopic endometrium of women with endometriosis is different from that of healthy women in terms of stem cell content, hormone sensitivity, cellular proliferation, adhesion, angiogenesis, and immunity. Progesterone resistance and defective endometrial receptivity impair the processes of implantation, decidualization, and placentation in women with endometriosis [21,22].

We did not find a higher rate of late pregnancy/obstetric complications and neonatal complications in women with small endome- triomas. Benaglia et al. [9] reported that late pregnancy/obstetric and neonatal outcomes did not differ in women with small endometriomas (the mean diameter was $22 \mathrm{~mm}$ in their study) compared with women without endometriomas. Another study conducted in Japan likewise showed no relationship between endometriosis and poor obstetric outcomes in women who did not conceive using IVF/ ICSI in order to exclude the negative effects of IVF on pregnancy outcomes [7].

However, recent systematic reviews and meta-analyses reported that obstetric complications such as preterm birth, placenta previa, cesarean section, and neonatal complications were more common in women with endometriosis $[6,19]$. The findings of previous studies have varied because all types of endometriosis were included. Moreover, earlier studies were not adjusted for confounders such as maternal age, poor obstetric history, parity, BMI, chronic disease, and socioeconomic status. The diagnosis of endometriosis was not uniform; in small studies, the diagnosis was made surgically, but in epidemiological studies the diagnosis was made using International Classification of Diseases codes, which have the potential of misclassification. The control groups were also heterogeneous in prior studies.

There are some limitations of this study; first, it was retrospective, which may have reduced the reliability of the findings. Second, the study size was relatively small. Third, we did not perform laparoscopy to confirm the endometriosis diagnosis histologically; instead, the endometriosis diagnosis was made by ultrasonography, and the patients were suspected to have endometrioma. However, the cases were small endometriomas and there was no need to perform surgery in order to improve pregnancy outcomes in these patients. Furthermore, we did not want to affect ovarian reserve negatively and increase patients' surgical and anesthetic risks. Fourth, the control group also did not undergo laparoscopy, so we cannot totally exclude the possibility that some women in the control group may have had mild endometriosis.

In conclusion, we showed that women with small endometriomas had higher rates of biochemical pregnancy and lower rates of clinical pregnancy and live birth; however, obstetric and neonatal complications did not differ between the groups. The findings suggest that women with endometriomas are more prone to early pregnancy complications than women with unexplained and tubal factor infertility. Women with small endometriomas undergoing ART should be counseled about these adverse aspects of their prognosis. Further prospective studies with larger groups are needed.

\section{Conflict of interest}

No potential conflict of interest relevant to this article was reported. 


\section{ORCID}

\section{Fatma Ferda Verit \\ https://orcid.org/0000-0002-7104-4532 Ayse Seyma Ozsuer Kucukakca https://orcid.org/0000-0002-7464-0591}

\section{Author contributions}

Conceptualization, Data curation, Formal analysis, Methodology, Project administration, Writing-original draft, review, \& editing: all authors.

\section{References}

1. Macer ML, Taylor HS. Endometriosis and infertility: a review of the pathogenesis and treatment of endometriosis-associated infertility. Obstet Gynecol Clin North Am 2012;39:535-49.

2. Leone Roberti Maggiore U, Ferrero S, Mangili G, Bergamini A, Inversetti A, Giorgione $V$, et al. A systematic review on endometriosis during pregnancy: diagnosis, misdiagnosis, complications and outcomes. Hum Reprod Update 2016;22:70-103.

3. Nisolle M, Casanas-Roux F, Donnez J. Immunohistochemical analysis of proliferative activity and steroid receptor expression in peritoneal and ovarian endometriosis. Fertil Steril 1997;68:912-9.

4. Barnhart K, Dunsmoor-Su R, Coutifaris C. Effect of endometriosis on in vitro fertilization. Fertil Steril 2002;77:1148-55.

5. Zullo F, Spagnolo E, Saccone G, Acunzo M, Xodo S, Ceccaroni M, et al. Endometriosis and obstetrics complications: a systematic review and meta-analysis. Fertil Steril 2017;108:667-72.

6. Lalani S, Choudhry AJ, Firth B, Bacal V, Walker M, Wen SW, et al. Endometriosis and adverse maternal, fetal and neonatal outcomes, a systematic review and meta-analysis. Hum Reprod 2018;33:1854-65.

7. Mekaru K, Masamoto H, Sugiyama H, Asato K, Heshiki C, Kinjyo T, et al. Endometriosis and pregnancy outcome: are pregnancies complicated by endometriosis a high-risk group? Eur J Obstet Gynecol Reprod Biol 2014;172:36-9.

8. Kortelahti M, Anttila MA, Hippelainen MI, Heinonen ST. Obstetric outcome in women with endometriosis: a matched case-control study. Gynecol Obstet Invest 2003;56:207-12.

9. Benaglia L, Bermejo A, Somigliana E, Scarduelli C, Ragni G, Fedele $L$, et al. Pregnancy outcome in women with endometriomas achieving pregnancy through IVF. Hum Reprod 2012;27:1663-7.
10. Fernando S, Breheny S, Jaques AM, Halliday JL, Baker G, Healy D. Preterm birth, ovarian endometriomata, and assisted reproduction technologies. Fertil Steril 2009;91:325-30.

11. Savelli L. Transvaginal sonography for the assessment of ovarian and pelvic endometriosis: how deep is our understanding? Ultrasound Obstet Gynecol 2009;33:497-501.

12. Dong $X$, Liao $X$, Wang $R$, Zhang $H$. The impact of endometriosis on IVF/ICSI outcomes. Int J Clin Exp Pathol 2013;6:1911-8.

13. Hamdan M, Dunselman G, Li TC, Cheong Y. The impact of endometrioma on IVF/ICSI outcomes: a systematic review and meta-analysis. Hum Reprod Update 2015;21:809-25.

14. Jacques M, Freour T, Barriere P, Ploteau S. Adverse pregnancy and neo-natal outcomes after assisted reproductive treatment in patients with pelvic endometriosis: a case-control study. Reprod Biomed Online 2016;32:626-34.

15. Rossi AC, Prefumo F. The effects of surgery for endometriosis on pregnancy outcomes following in vitro fertilization and embryo transfer: a systematic review and meta-analysis. Arch Gynecol Obstet 2016;294:647-55.

16. Harb HM, Gallos ID, Chu J, Harb M, Coomarasamy A. The effect of endometriosis on in vitro fertilisation outcome: a systematic review and meta-analysis. BJOG 2013;120:1308-20.

17. Omland AK, Abyholm T, Fedorcsak P, Ertzeid G, Oldereid NB, Bjercke $S$, et al. Pregnancy outcome after IVF and ICSI in unexplained, endometriosis-associated and tubal factor infertility. Hum Reprod 2005;20:722-7.

18. Ding GL, Chen XJ, Luo Q, Dong MY, Wang N, Huang HF. Attenuated oocyte fertilization and embryo development associated with altered growth factor/signal transduction induced by endometriotic peritoneal fluid. Fertil Steril 2010;93:2538-44.

19. Horton J, Sterrenburg M, Lane S, Maheshwari A, Li TC, Cheong Y. Reproductive, obstetric, and perinatal outcomes of women with adenomyosis and endometriosis: a systematic review and meta-analysis. Hum Reprod Update 2019;25:592-632.

20. Bedaiwy MA, Falcone T. Peritoneal fluid environment in endometriosis: clinicopathological implications. Minerva Ginecol 2003; 55:333-45.

21. Vercellini P, Vigano P, Somigliana E, Fedele L. Endometriosis: pathogenesis and treatment. Nat Rev Endocrinol 2014;10:261-75.

22. Benagiano G, Brosens I, Habiba M. Structural and molecular features of the endomyometrium in endometriosis and adenomyosis. Hum Reprod Update 2014;20:386-402. 